\title{
Coinfection of Tuberculosis Pneumonia and COVID-19 in a Patient Vaccinated with Bacille Calmette-Guérin (BCG): Case Report
}

\author{
Michelangelo Luciani ${ }^{1}$ (D) Enrico Bentivegna ${ }^{1} \cdot$ Valerio Spuntarelli $^{2} \cdot$ Piera Amoriello Lamberti $^{1}$. \\ Ludovica Guerritore $^{1}$. Dario Chiappino ${ }^{1} \cdot$ Gabriele Nalli $^{1}$ - Maria Proietta ${ }^{1}$. Flavia Del Porto ${ }^{1} \cdot$ Paolo Martelletti $^{2}$. \\ Giorgio Sesti ${ }^{1}$
}

Accepted: 15 October 2020 / Published online: 17 October 2020

(C) Springer Nature Switzerland AG 2020

\begin{abstract}
COVID-19 is a respiratory tract infection caused by the new coronavirus SARS-COV2 that can be complicated by acute distress respiratory syndrome and multiorgan failure. In light of the high rate of mortality associated with COVID-19, pharmacological and non-pharmacological strategies to prevent the infection are currently being tested. Among non-pharmacological preventive measures, vaccines represent one of the main resources for public health. It has been suggested that Bacille Calmette-Guérin (BCG) vaccine may protect individuals against infection from COVID-19 virus, and two clinical trials addressing this question are underway. Here, we report the case of a 32-year-old woman, vaccinated with BCG when she was 1 year old, who was diagnosed with apical tuberculous pneumonia of the right lung along with COVID 19 pneumonia.
\end{abstract}

Keywords Covid-19 $\cdot$ SARS-CoV-2 $\cdot$ Tuberculosis $\cdot$ Pneumonia $\cdot$ Immunity $\cdot$ BCG

\section{Introduction}

COVID-19 is a respiratory tract infection caused by the coronavirus SARS-CoV-2. This virus was first reported in Wuhan (China) and rapidly spread worldwide causing a pandemic [1] with over 300,000 deaths up until July 2020 [2]. Nonpharmacological strategies have been adopted to prevent infection diffusion, including quarantine, social distancing, and personal protective equipment. COVID-19 clinical features include a spectrum of manifestations, ranging from absence of symptoms, gastro-intestinal disorders, and flu-like

Paolo Martelletti and Giorgio Sesti contributed equally to this work.

This article is part of the Topical Collection on Covid-19

Michelangelo Luciani

michelangelo.luciani@uniroma1.it

1 Faculty of Medicine and Psychology, Department of Clinical and Molecular Medicine, Internal Medicine Unit, Sant'Andrea University Hospital, Sapienza University, Rome, Italy

2 Faculty of Medicine and Psychology, Emergency Medicine CoViD-19 Unit, Sant'Andrea University Hospital, Sapienza University, Rome, Italy symptoms including fever, cough, and astenia [3]. In older patients with comorbidities, SARS-CoV-2 leads to interstitial pneumonia, acute respiratory distress syndrome (ARDS), multiorgan failure, and, eventually, death [4]. COVID-19 can be complicated by bacterial superinfections. Antibiotics, immunosuppressants, low weight heparin, and antiviral drugs have been used to treat patients $[3,4]$. Among preventive measures, vaccines represent a major resource for public health, and it has been suggested that Bacille CalmetteGuérin (BCG) vaccine may protect individuals against COVID-19 infection: two clinical trials addressing this hypothesis are underway [5].

Here, we report the case of a 32-old-year woman, vaccinated with BCG, simultaneously affected by COVID-19 and tuberculous pneumonia.

\section{Case Description}

A 32-year-old woman, with history of BCG vaccination at the age of one, was hospitalized on 29 March 2020 with a 3-weeklong high fever and muscle pain. The patient had a positive epidemiological link with travel to Milan, an area in Italy 
severely hit by COVID-19, and was treated with amoxicillin/ clavulanic acid $1000 \mathrm{mg} / \mathrm{BID}$ and paracetamol $1000 \mathrm{mg} /$ day.

On day one of hospitalization, the first nasopharyngeal swab RNA test for SARS-CoV2 genes resulted undetermined, while two subsequent tests were negative on day two and day three, respectively. High-resolution chest computer tomography (HRCT) revealed parenchymal consolidation of $54 \times$ $25 \mathrm{~mm}$, with associated air bronchogram, at the apical segment of the right upper lobe (RUL) (Fig. 1). Other consolidation areas with the same feature were located in the posterior segment of the same lobe in the parascissural site, in the middle lobe in the paramediastinal site, and contralaterally to the anterior segment of the left upper lobe (LUL) in the subpleural area. Large right pleural effusion leading to atelectasis of the right lower lobe (RLL) was also detected.

Blood tests revealed lymphopenia $\left(900 / \mathrm{mm}^{3}\right.$ and $15.6 \%$ of leukocyte formula) and increase in high sensitive $\mathrm{C}$-reactive protein (hsCRP $18.08 \mathrm{mg} / \mathrm{dL}$ ) and procalcitonin (PCT $0.43 \mathrm{ng} / \mathrm{mL})$, fibrinogen, $(882 \mathrm{mg} / \mathrm{dL})$ and d-dimer $(622 \mathrm{ng} /$ $\mathrm{mL})$. Antibiotic therapy was started with clarithromycin $500 \mathrm{mg} / \mathrm{BID}$ together with piperacillin/tazobactam $4.5 \mathrm{~g} / \mathrm{TID}$.

On day four, a new nasopharyngeal swab RNA test for SARS-CoV-2 genes resulted positive, while both IgG and IgM antibodies assays were negative. The patient was transferred to a dedicated COVID-19 unit, where hydroxychloroquine and antiviral therapy with lopinavir/ ritonavir were initiated. A first measurement of

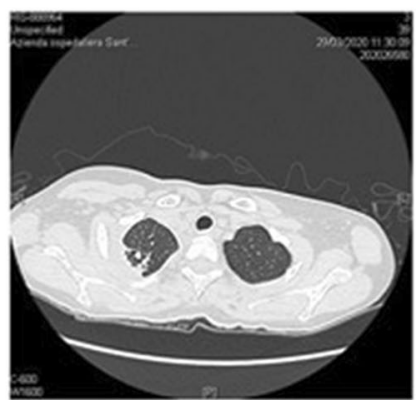

$1 \mathrm{a}$

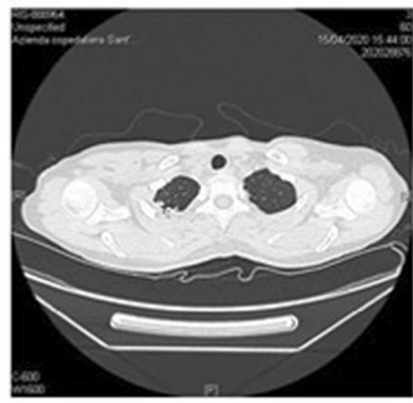

$2 a$

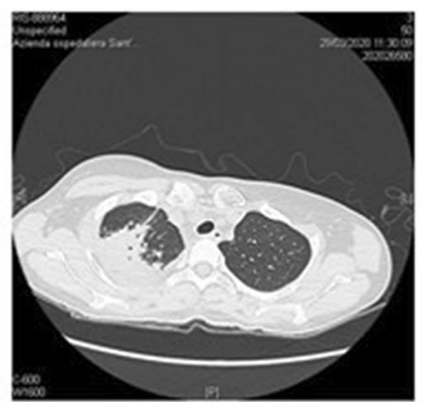

1b

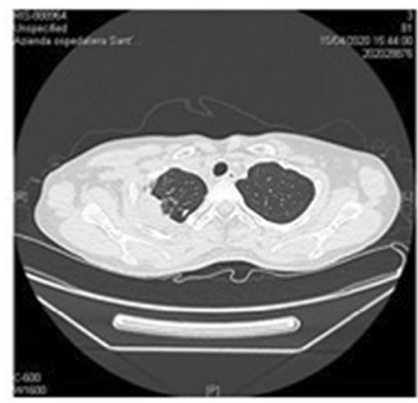

2b

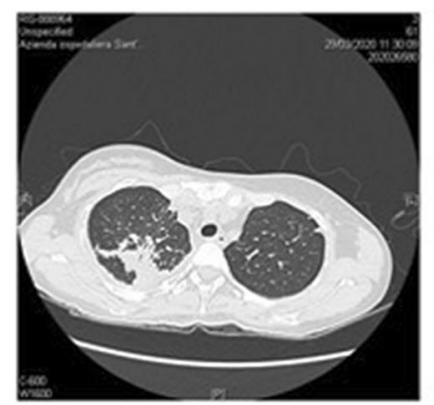

$1 \mathrm{C}$

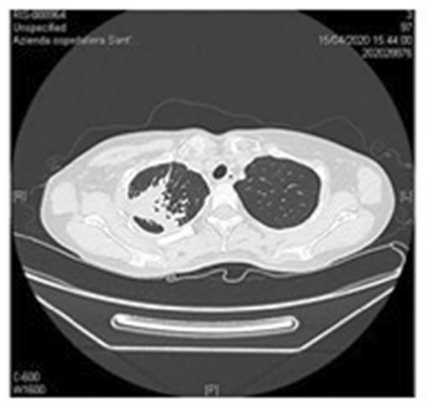

2c
QuantiFERON® gave an indeterminate result with the indication to repeat the exam because of insufficient production of gamma interferon upon stimulation with mitogen. HIV test was negative. QuantiFERON® and HIV tests were performed in the suspicion of tuberculous disease: therefore, both the location of the lesion and the patient's nationality had made way for a possible tuberculous infection obviously facilitated by a state of immunodeficiency.

On day seven, linezolid $500 \mathrm{mg} / \mathrm{BID}$ was introduced by replacing the previous antibiotic therapies due to the lack of clinical improvement. Thoracentesis was performed with the removal of $1 \mathrm{~L}$ of citrine yellow liquid. The chemical-physical and cytological analyses of the pleural fluid were consistent with exudative effusion, while culture tests for common germs and real-time polymerase chain reaction assay (RT-PCR) of Bacillus of Koch (KB) were both negative. Due to rapid relapse of large pleural effusion, pleural drainage with pigtail catheter was inserted. A second assay for the QuantiFERON® gave an indeterminate result. Due to the uncertain interpretation of chest CT images despite the reduction of pleural effusion, a lung CT biopsy procedure was performed with histological examination showing multiple areas of caseous necrosis delimited by a fibrous wall and giant multinucleated cells suggestive for tuberculosis infection. RT-PCR performed on histological samples resulted positive for KB.

Between day nine and day twelve, the patient repeated three nasopharyngeal swab RNA tests for SARS-CoV-2
Fig. 1 First HRCT showing parenchymal consolidation with air bronchogram at the right apical segment (1A). Other consolidation areas were located in the posterior segment of the RUL and contralaterally (1B and $1 \mathrm{C}$ ). Large right pleural effusion leading to

atelectasis of RLL was also detected (1D). Second HRCT performed after 2 weeks showing reduction of parenchymal consolidation (2A, B, and C) and resolution of pleural effusion (2D) 
genes which were negative, while clinical conditions improved. The antiviral therapy was first stopped followed by the hydroxychloroquine treatment. After discharge, the patient observed a 14-day quarantine period, and due to positive tests for tuberculosis, a combined therapy with isoniazid, rifampicin, pyrazinamide, and ethambutol was introduced. No oxygen therapy was required during hospitalization.

\section{Discussion}

To our knowledge, this is the first report in the literature of acute tuberculosis infection superimposed on COVID-19 pneumonia. The association of these two infections has been previously described in 3 patients, but none of them developed an acute tuberculous disease, even though two of them were under treatment with quadruple anti-tuberculosis therapy, which was able to prevent the reactivation of the disease [6].

By meticulously and thoroughly analyzing the patient's anamnestic history, it was not possible to detect previous clinical manifestations that suggest a long-standing state of immunodeficiency. In fact, both the patient in question and her close relatives, when repeatedly questioned about possible diseases and previous manifestations, denied any compromise of the state of health even of a mild nature. This led to believe that the tuberculous infection, which until then had never manifested itself, had become infected with the coronavirus- 19 .

Interestingly, our patient had a history of $\mathrm{BCG}$ vaccination, a life-attenuated vaccine for tuberculosis that contains a weakened strain of Mycobacterium bovis. It is given to children in their first year of life in several countries, conferring protection against active tuberculosis in about $60 \%$ of treated individuals [7]. BCG increases immune response also to other pathogens, conferring a nonspecific protection against a wide range of bacterial, protozoa, and viral infections, throughout the activation of the innate immune system [8]. It also induces the innate immune system to develop a "memory", which is called "trained immunity," through epigenetic reprogramming of monocytes, macrophages, and natural killer cells $[9,10]$. Trained immunity induced by BCG may affect different microorganisms other than Mycobacterium tuberculosis. In a recent study carried out in healthy volunteers, vaccination with BCG was shown to reduce viremia in response to the yellow fever vaccine (live attenuated vaccine) [11]. This response was associated with epigenetic changes in monocytes which were related to a better antiviral response [12]. The broad immunomodulatory capacity of BCG on respiratory tract pathogens was supported by a study in Guinea-Bissau showing that BCG reduced the incidence of respiratory syncytial virus infection [13], while a similar protective effect of BCG on infections respiratory tract has been reported in elderly subjects in Indonesia [14].
It has been hypothesized that trained immunity can also exert protective actions against COVID-19. In countries that do not have a universal vaccination policy against the BCG, such as Italy and the USA, a higher mortality associated with COVID-19 has been reported as compared with nations with long-standing universal vaccination policies with BCG, such as South Korea and Japan [15].

BCG confers immunity against Mycobacterium tuberculosis for about 10 years although some reports describe a duration of immunity up to 20 years [16].

This case suggests that in some individuals, the immune response to $\mathrm{BCG}$ vaccination may be defective as supported by the findings that results of QuantiFERON® assay were indeterminate indicating a condition of nonresponsiveness of $\mathrm{T}$ lymphocytes to the antigenic stimuli. Moreover, the effect of the BCG vaccination on trained immunity seems to be limited to 1 year in most cases [11]. Thus, it is conceivable that in our patient, BCG vaccination did not induce a trained immunity capable to provide protection against either tuberculosis infection or COVID19 infections. Indeed, the patient's immune system was unable to react to Mycobacterium tuberculosis infection by generating a latent infection that subsequently was flared up under a stressful condition induced by viral superinfection with coronavirus-19 [17].

Furthermore, the double evaluation of QuantiFERON®, which proved twice to be indeterminate, could be the result of a momentary state of immunosuppression which subsequently allowed the occurrence of this combined bacterial and viral "superinfection."

Clinical trials are underway to investigate the effect of BCG vaccination to protect the elderly individuals from infections and to explore the possibility to prevent serious COVID19 infection in healthcare professionals. The current case report, although representing only a single case and therefore not sufficient to support any hypothesis, certainly contributes to discussions against the possibility that $\mathrm{BCG}$ vaccination exerts long-lasting protection from coronavirus-19 infection and highlights the importance of further research aimed to explore the role of trained immunity induced by the BCG vaccine in COVID-19 management [18].

\footnotetext{
Authors' Contributions Dr. Luciani participated in article preparation. All authors materially participated in the research. All authors participated in data collection. All authors have approved the final article.
}

\section{Compliance with Ethical Standards}

Conflict of Interest The authors declare that they have no conflict of interest.

Ethical Approval Prot. n. 52 SA_2020 del Registro Pareri Rif. CE 5773_2020 
Informed Consent Written informed consent was obtained by patient for publication of this case report and accompanying images.

Registration of Research Studies NA.

Guarantors Prof. Martelletti Paolo, MD and Prof. Sesti Giorgio, MD

\section{References}

1. Zhu N, Zhang D, Wang W, et al. A novel coronavirus from patients with pneumonia in China. N Engl J Med. 2020;382(8):727-33.

2. COVID-19 Dashboard by the Center for Systems Science and Engineering (CSSE) at Johns Hopkins University. https:// coronavirus.jhu.edu/map.html Retrieved 15 May 2020.

3. Pascarella G, Strumia A, Piliego C, Bruno F, Del Buono R, Costa F, et al. COVID-19 diagnosis and management: a comprehensive review. J Intern Med. 2020;288(2):192-206. https://doi.org/10.1111/ joim.13091 Epub 2020 May 13.

4. Bentivegna E, Luciani M, Spuntarelli V, Speranza ML, Guerritore L, Sentimentale A, et al. Extremely severe case of COVID-19 pneumonia recovered despite bad prognostic indicators: a didactic report. SN Compr Clin Med. 2020;29:1-4. https://doi.org/10.1007/ s42399-020-00383-0 Epub ahead of print.

5. O'Neill LAJ, Netea MG. BCG-induced trained immunity: can it offer protection against COVID-19? Nat Rev Immunol. 2020;20(6):335-7. https://doi.org/10.1038/s41577-020-0337-y.

6. He G, Wu J, Shi J, Dai J, Gamber M, Jiang X, Sun W, Cai J. COVID-19 in tuberculosis patients: a report of three cases. J Med Virol. 2020, 28:https://doi.org/10.1002/jmv.25943.

7. Roy A, Eisenhut M, Harris RJ, Rodrigues LC, Sridhar S, Habermann S, et al. Effect of BCG vaccination against Mycobacterium tuberculosis infection in children: systematic review and meta-analysis. BMJ. 2014;349:g4643. https://doi.org/ 10.1136/bmj.g4643.

8. Shann F. The non-specific effects of vaccines. Arch Dis Child. 2010;95(9):662-7. https://doi.org/10.1136/adc.2009.157537.

9. Kleinnijenhuis J, Quintin J, Preijers F, Joosten LA, Ifrim DC, Saeed $\mathrm{S}$, et al. Bacille Calmette-Guerin induces NOD2-dependent nonspecific protection from reinfection via epigenetic reprogramming of monocytes. Proc Natl Acad Sci U S A. 2012;109(43):17537-42. https://doi.org/10.1073/pnas.1202870109.
10. Kleinnijenhuis J, Quintin J, Preijers F, Joosten LA, Jacobs C, Xavier RJ, et al. BCG-induced trained immunity in NK cells: role for non-specific protection to infection. Clin Immunol. 2014;155(2):213-9. https://doi.org/10.1016/j.clim.2014.10.005.

11. Arts RJW, Moorlag SJCFM, Novakovic B, Li Y, Wang SY, Oosting $\mathrm{M}$, et al. BCG vaccination protects against experimental viral infection in humans through the induction of cytokines associated with trained immunity. Cell Host Microbe. 2018;23(1):89 100. e5. https://doi.org/10.1016/j.chom.2017.12.010.

12. Covián C, Retamal-Díaz A, Bueno SM, Kalergis AM. Could BCG vaccination induce protective trained immunity for SARS-CoV-2? Front Immunol. 2020;11:970. https://doi.org/10.3389/fimmu.2020. 00970.

13. Stensballe LG, Nante E, Jensen IP, Kofoed PE, Poulsen A, Jensen $\mathrm{H}$, et al. Acute lower respiratory tract infections and respiratory syncytial virus in infants in Guinea-Bissau: a beneficial effect of BCG vaccination for girls community based case-control study. Vaccine. 2005 Jan 26;23(10):1251-7. https://doi.org/10.1016/j. vaccine.2004.09.006.

14. Wardhana, Datau EA, Sultana A, Mandang VV, Jim E. The efficacy of Bacillus Calmette-Guerin vaccinations for the prevention of acute upper respiratory tract infection in the elderly. Acta Med Indones 2011;43(3):185-190.

15. Miller A, et al. Correlation between universal BCG vaccination policy and reduced morbidity and mortality for COVID- 19 . medRxiv 2020.03.24.20042937; https://doi.org/10.1101/2020.03. 24.20042937

16. Martin C, Aguilo N, Gonzalo-Asensio J. Vacunación frente a tuberculosis. Enferm Infecc Microbiol Clin. 2017;36:648-56. https:// doi.org/10.1016/j.eimc.2018.02.006.

17. Bentivegna E, Sentimentale A, Luciani M, Speranza ML, Guerritore L, Martelletti P. New IgM seroconversion and positive RT-PCR test after exposure to the virus in recovered COVID-19 patient. J Med Virol. 2020. https://doi.org/10.1002/jmv.26160.

18. Hegarty PK, Sfakianos JP, Giannarini G, DiNardo AR, Kamat AM. COVID-19 and Bacillus Calmette-Guérin: what is the link? Eur Urol Oncol. 2020;3(3):259-61. https://doi.org/10.1016/j.euo.2020. 04.001 .

Publisher's Note Springer Nature remains neutral with regard to jurisdictional claims in published maps and institutional affiliations. 